\title{
Lagrangian and energy forms for retrieving the impulse response of the Earth due to random electromagnetic forcing
}

\author{
Evert Slob ${ }^{1, *}$ and Chester J. Weiss ${ }^{2, \dagger}$ \\ ${ }^{1}$ Department of Geotechnology, Delft University of Technology, Post Office Box 5048, NL-2600 GA Delft, The Netherlands \\ ${ }^{2}$ Department of Geosciences, Virginia Tech, Blacksburg, Virginia 24061, USA
}

(Received 7 January 2011; revised manuscript received 5 July 2011; published 10 August 2011)

\begin{abstract}
We distinguish between trivial and nontrivial differences in retrieving the real or imaginary parts of the Green's function. Trivial differences come from different Green's function definitions. The energy and Lagrangian forms constitute nontrivial differences. Magnetic noise sources suffice to extract the quasistatic electromagnetic-field Earth impulse response in the Lagrangian form. This is of interest for Earth subsurface imaging. A numerical example demonstrates that all source vector components are necessary to extract a single-field vector component.
\end{abstract}

DOI: 10.1103/PhysRevE.84.027601

\section{INTRODUCTION}

The extraction of Green's functions by correlations of recorded field fluctuations generated by noise sources, or other types of uncorrelated sources, has been studied widely in acoustics, seismology, and electromagnetics. Using an energy form of a time-correlation-type reciprocity relation, general field formulations based on first-order [1] and second-order [2] differential equations exist. In these formulations, different definitions of Green's functions are used. This difference causes the relations based on first-order equations to retrieve the real part of the Green's functions, whereas the imaginary part of the Green's functions are retrieved in relations based on the second-order equations. This is not a necessary difference, but the difference has occurred due to different Green's function definitions.

Recently Shamsalsadati and Weiss [3] published a new magnetic field correlation version of Green's function retrieval. The basic relation used in [3] is the electromagnetic example of the general relation given in [4], which is based on the Lagrangian form of a correlation-type reciprocity theorem. Shamsalsadati and Weiss did not recognize their novelty in [3], but it is this choice that defines the required correlation strength of the noise sources for Green's function retrieval. It has been shown previously that the energy form leads to electric field and magnetic field Green's functions that can be retrieved by noise sources whose correlation strength is proportional to the real parts of the electric and magnetic conductivity $[5,6]$. The energy form can be understood from the fluctuation-dissipation theorem when thermal noise is the driving mechanism [7], while the Lagrangian form cannot. Below we investigate how the Lagrangian form leads to the formulation derived in [3] and to a similar relation for the retrieval of the electric field impulse response.

In their final Green's function retrieval formulation, Shamsalsadati and Weiss state that a single source vector component is sufficient to retrieve the Green's function. Their formulation is not explicit on how that should work. They include noise sources without specifying the necessary constraints on the noise sources. They do not show how the Green's function

\footnotetext{
*e.c.slob@tudelft.nl

${ }^{\dagger}$ cjweiss@vt.edu
}

PACS number(s): 41.90.+e, 91.25.Qi, 05.40.Ca, 82.56.Lz

emerges as the expectation value of the correlation of noise magnetic field recordings taken at two locations. We derive a formulation showing explicitly how receiver and source vector components play a role in Green's function retrieval and what the constraints on the noise sources are.

In their derivation, Shamsalsadati and Weiss simplify the Green's function retrieval relation. They neglect the integral containing the inner product of the curl of magnetic fields based on the argument that they work in the quasistatic limit where $\omega \varepsilon \ll \sigma$. They are interested in the quasistatic limit, because the controlled source electromagnetic method uses frequencies in the order of $1 \mathrm{~Hz}$ to explore crustal-scale targets such as hydrocarbon reservoirs, as well as tectonic and sedimentary basin architecture. We use a homogeneous space example to show the validity of their argument, which also demonstrates the incorrectness of using aligned noise source dipoles to extract the Green's function.

\section{INTEGRAL REPRESENTATIONS FOR THE MAGNETIC FIELD}

We use vector notation similar to the one used in [3] and give two different reciprocity relations based on second-order equations satisfied by the magnetic fields. We use $\boldsymbol{h}$ to denote the magnetic field vector. The two subscripts $A$ and $B$ denote the two possibly different states; $\eta=\sigma^{e}+i \omega \varepsilon, \zeta=\sigma^{m}+$ $i \omega \mu$ denote the generalized electric and magnetic conductivity, respectively, in which $\sigma^{e}, \sigma^{m}$ are the electric and magnetic conductivity, while $\varepsilon, \mu$ denote the electric permittivity and magnetic permeability and $\omega$ is radial frequency; $\boldsymbol{f}=-\boldsymbol{j}^{m}+$ $\boldsymbol{\nabla} \times\left(\eta^{-1} \boldsymbol{j}^{e}\right)$ is the source vector with $\boldsymbol{j}^{e}, \boldsymbol{j}^{m}$ the sources of electric and magnetic current type, respectively. We can then write the local form of field interactions using a second-order Maxwell equation for the magnetic fields as

$$
\begin{gathered}
\boldsymbol{h}_{B}^{*} \cdot \nabla \times \frac{\eta^{*}}{|\eta|^{2}} \nabla \times \boldsymbol{h}_{A}+\zeta \boldsymbol{h}_{A} \cdot \boldsymbol{h}_{B}^{*}=\boldsymbol{h}_{B}^{*} \cdot \boldsymbol{f}_{A}, \\
\boldsymbol{h}_{A} \cdot \nabla \times \frac{\eta}{|\eta|^{2}} \nabla \times \boldsymbol{h}_{B}^{*}+\zeta^{*} \boldsymbol{h}_{A} \cdot \boldsymbol{h}_{B}^{*}=\boldsymbol{h}_{A} \cdot \boldsymbol{f}_{B}^{*} .
\end{gathered}
$$

Both equations can be integrated over an arbitrary domain $\mathbb{D}$, and in the part containing the curl operators, integration by parts can be used together with Gauss's integral theorem. 
Then we can add or subtract the results, leading to two different reciprocity relations of the time correlation type,

$$
\begin{aligned}
\oint_{\partial \mathbb{D}} & \left(\frac{\eta^{*}}{|\eta|^{2}}\left(\nabla \times \boldsymbol{h}_{A}\right) \times \boldsymbol{h}_{B}^{*} \pm \frac{\eta}{|\eta|^{2}}\left(\nabla \times \boldsymbol{h}_{B}^{*}\right) \times \boldsymbol{h}_{A}\right) \cdot \boldsymbol{n} \mathrm{d}^{2} \boldsymbol{r} \\
- & \int_{\mathbb{D}} \frac{\eta \pm \eta^{*}}{|\eta|^{2}}\left(\nabla \times \boldsymbol{h}_{A}\right) \cdot\left(\boldsymbol{\nabla} \times \boldsymbol{h}_{B}^{*}\right) \mathrm{d}^{3} \boldsymbol{r} \\
& +\int_{\mathbb{D}}\left(\zeta \pm \zeta^{*}\right) \boldsymbol{h}_{A} \cdot \boldsymbol{h}_{B}^{*} \mathrm{~d}^{3} \boldsymbol{r}=\int_{\mathbb{D}}\left(\boldsymbol{h}_{B}^{*} \cdot \boldsymbol{f}_{A} \pm \boldsymbol{h}_{A} \cdot \boldsymbol{f}_{B}^{*}\right) \mathrm{d}^{3} \boldsymbol{r} .
\end{aligned}
$$

When we use the plus sign for the \pm options in Eq. (3), we obtain the reciprocity relation of the time correlation type on which the work of Wapenaar [1,8] is based and which is similar to the electromagnetic formulations derived in [6]. The only difference is that here we obtain it starting with a second-order equation, whereas in $[1,6]$ the point of departure is a coupled set of first-order equations. This difference is of course irrelevant. Snieder works with a minus sign in $[9,10]$ yet uses the energy form [plus sign in Eq. (3)]. This is because he defines his second-order starting equation differently. The electromagnetic equivalent of Snieder's scalar formulation (see, e.g., [11]), is obtained by introducing an auxiliary form. We replace $\eta$ by $i \omega \hat{\varepsilon}$ and $\zeta$ by $i \omega \hat{\mu}$ in Eq. (3) and define the new source as $\hat{\boldsymbol{f}}=i \omega \boldsymbol{f}$ to obtain

$$
\begin{aligned}
\oint_{\partial \mathbb{D}} & \left(\frac{\hat{\varepsilon}^{*}}{|\hat{\varepsilon}|^{2}}\left(\nabla \times \boldsymbol{h}_{A}\right) \times \boldsymbol{h}_{B}^{*} \mp \frac{\hat{\varepsilon}}{|\hat{\varepsilon}|^{2}}\left(\nabla \times \boldsymbol{h}_{B}^{*}\right) \times \boldsymbol{h}_{A}\right) \cdot \boldsymbol{n} \mathrm{d}^{2} \boldsymbol{r} \\
+ & \int_{\mathbb{D}} \frac{\hat{\varepsilon} \mp \hat{\varepsilon}^{*}}{|\hat{\varepsilon}|^{2}}\left(\nabla \times \boldsymbol{h}_{A}\right) \cdot\left(\nabla \times \boldsymbol{h}_{B}^{*}\right) \mathrm{d}^{3} \boldsymbol{r} \\
& -\omega^{2} \int_{\mathbb{D}}\left(\hat{\mu} \mp \hat{\mu}^{*}\right) \boldsymbol{h}_{A} \cdot \boldsymbol{h}_{B}^{*} \mathrm{~d}^{3} \boldsymbol{r}=\int_{\mathbb{D}}\left(\boldsymbol{h}_{B}^{*} \cdot \hat{\boldsymbol{f}}_{A} \mp \boldsymbol{h}_{A} \cdot \hat{\boldsymbol{f}}_{B}^{*}\right) \mathrm{d}^{3} \boldsymbol{r} .
\end{aligned}
$$

Now we see that choosing the minus sign for all \pm options in Eq. (4) is the energy form. Notice that the definitions of the fields did not change, only those of the sources, which means that also the Green's functions are defined differently in the auxiliary form of Eq. (4) than in the traditional form of Eq. (3). This demonstrates that the choice of the Green's function determines whether addition or subtraction of the interaction equations is necessary to obtain an energy form as the basis for Green's function extraction.

When we use the minus sign for all \pm options in Eq. (3), we arrive at the result of Shamsalsadati and Weiss given in Eq. (15) of [3]. This is under the condition that the resulting boundary integral vanishes, which is their Eq. (16). Choosing the minus sign for all options \pm in Eq. (3) leads to the Langrangian form of reciprocity of the time correlation type, which was new in their publication, and it was independently generalized in [4]. The distinction between the energy form and the Lagrangian form of time-correlation-type reciprocity relations is a fundamental difference as a basis for Green's function retrieval. The energy form was used by Rytov and co-workers in the development of the theory of thermal electromagnetic radiation from absorbing media [7], which is a direct result of the application of the fluctuation-dissipation theorem. The Langrangian form is new and leads to noise sources different than the Rytov theory, which is shown in the next section.

\section{REPRESENTATION THEOREMS AND GREEN'S FUNCTIONS}

Here we investigate only the Lagrangian form of Eq. (3), and hence we select the minus sign. By taking a dissipative medium of infinite extent, the boundary integral in the lefthand side of Eq. (3) vanishes, and if we restrict our analysis to frequencies of which $\sigma^{e} \gg \omega \varepsilon$, the second integral in the lefthand side of Eq. (3) can be neglected. We take the sources to be Dirac sources as $\boldsymbol{f}_{A}(\boldsymbol{r})=\boldsymbol{s}_{A} \delta\left(\boldsymbol{r}-\boldsymbol{r}_{A}\right)$, where $\boldsymbol{s}_{A}$ denotes the real-valued unit vector representing the source direction in $\boldsymbol{r}_{A}$, and a similar choice is made for $f_{B}$. Then the fields become Green's function type magnetic field vectors, $\overline{\boldsymbol{h}}_{A}(\boldsymbol{r})$, which can be written in terms of the Green's tensor function, $G_{j p}$ as $\overline{\boldsymbol{h}}_{A}(\boldsymbol{r})=G_{j p}\left(\boldsymbol{r}, \boldsymbol{r}_{A}\right) s_{p}\left(\boldsymbol{r}_{A}\right)$, which is written in subscript notation for clarity, and the summation convention applies to repeated lowercase Latin subscripts. A similar expression can be written down for $\overline{\boldsymbol{h}}_{B}^{*}(\boldsymbol{r})$. We substitute these choices in Eq. (3) and find

$$
\boldsymbol{s}_{A} \cdot \overline{\boldsymbol{h}}_{B}^{*}\left(\boldsymbol{r}_{A}\right)-\boldsymbol{s}_{B} \cdot \overline{\boldsymbol{h}}_{A}\left(\boldsymbol{r}_{B}\right)=\int_{\mathbb{D}}\left(\zeta-\zeta^{*}\right) \overline{\boldsymbol{h}}_{B}^{*} \cdot \overline{\boldsymbol{h}}_{A} \mathrm{~d}^{3} \boldsymbol{r} .
$$

When we take $s_{A}=s_{B}$, Eq. (5) is the same equation as Eq. (20) in [3]. There Shamsalsadati and Weiss make a remark about evaluating the integral in the right-hand side of Eq. (5), while making use of reciprocity, and then turn to an equivalence statement. Assuming that the source vectors are equal, $\boldsymbol{s}_{A}=$ $\boldsymbol{s}_{B}$, with application of these two steps to the integrand in the right-hand side of Eq. (5), we can write the integrand as

$$
\begin{aligned}
G_{j p}^{*}\left(\boldsymbol{r}, \boldsymbol{r}_{B}\right) s_{p} G_{j q}\left(\boldsymbol{r}, \boldsymbol{r}_{A}\right) s_{q} & =s_{p} G_{p j}^{*}\left(\boldsymbol{r}_{B}, \boldsymbol{r}\right) s_{q} G_{q j}\left(\boldsymbol{r}_{A}, \boldsymbol{r}\right) \\
& =s_{p} G_{p j}^{*}\left(\boldsymbol{r}_{B}, \boldsymbol{r}\right) s_{j} s_{q} G_{q m}\left(\boldsymbol{r}_{A}, \boldsymbol{r}\right) s_{m}
\end{aligned}
$$

where the fist step is a correct application of source-receiver reciprocity, but the second step can only be made when $s_{j} s_{m}=$ $\delta_{j m}$, which is in general contradiction with the assumption made beforehand that $s_{A}=s_{B}$. We conclude from this analysis that Eq. (23) in [3] is incorrect. The reason is that after reciprocity has been applied, the vectors $s$ represent receiver orientations and are completely independent from the required source orientations. Relation (5) is given in components by

$$
\operatorname{Im}\left\{G_{k r}\left(\boldsymbol{r}_{A}, \boldsymbol{r}_{B}\right)\right\}=-\operatorname{Im}\{\zeta\} \int_{\mathbb{D}} G_{k p}^{*}\left(\boldsymbol{r}_{B}, \boldsymbol{r}\right) G_{r p}\left(\boldsymbol{r}_{A}, \boldsymbol{r}\right) \mathrm{d}^{3} \boldsymbol{r} .
$$

Notice that the sum over the subscript ${ }_{p}$ remains, for which reason the result in [3] is incorrect because they assign the value 3 to all subscripts to select the vertical component. Equation (7) is an important correction over Eq. (25) in [3] and is used for a validating example. Equation (25) in [3] is given for noise sources, but the noise characteristics of these sources is not discussed or used there. Let us now choose space-time-dependent naturally occurring random sources $\boldsymbol{N}(\boldsymbol{r}, t)$, represented in the frequency domain as $\boldsymbol{n}(\boldsymbol{r}, \omega)$, and we can write the magnetic field vector generated by these noise sources and measured at $\boldsymbol{r}_{A}$ and $\boldsymbol{r}_{B}$, in subscript notation and in the frequency domain as $h_{j}\left(\boldsymbol{r}_{A}\right)=\int_{\boldsymbol{r} \in \mathbb{D}} G_{j p}\left(\boldsymbol{r}_{A}, \boldsymbol{r}\right) n_{p}(\boldsymbol{r}) \mathrm{d}^{3} \boldsymbol{r}$, and a similar relation for $h_{q}\left(\boldsymbol{r}_{B}\right)$. If the noise sources are uncorrelated and satisfy $\left\langle n_{p}(\boldsymbol{r}) n_{r}^{*}\left(\boldsymbol{r}^{\prime}\right)\right\rangle=\delta_{p r} \delta\left(\boldsymbol{r}-\boldsymbol{r}^{\prime}\right)|f(\omega)|^{2}$, 
where $\langle\cdot\rangle$ denotes the expectation value and the power spectrum $|f(\omega)|^{2}$ of the noise sources accounts for possible variable frequency dependence, the product $\left\langle h_{j}\left(\boldsymbol{r}_{A}\right) h_{q}^{*}\left(\boldsymbol{r}_{B}\right)\right\rangle$ is equal to $\int G_{j p}\left(\boldsymbol{r}_{A}, \boldsymbol{r}\right) G_{q p}^{*}\left(\boldsymbol{r}_{B}, \boldsymbol{r}\right) \mathrm{d}^{3} \boldsymbol{r}|f(\omega)|^{2}$. In that case, $\left\langle h_{j}\left(\boldsymbol{r}_{A}\right) h_{q}^{*}\left(\boldsymbol{r}_{B}\right)\right\rangle$ is equal to the integral in the right-hand side of Eq. (7), apart from the power spectrum of the noise, and hence we find the general expression for the Green's function as

$$
\operatorname{Im}\left\{G_{q j}\left(\boldsymbol{r}_{A}, \boldsymbol{r}_{B}\right)\right\}|f(\omega)|^{2}=-\operatorname{Im}\{\zeta\}\left\langle h_{j}\left(\boldsymbol{r}_{A}\right) h_{q}^{*}\left(\boldsymbol{r}_{B}\right)\right\rangle .
$$

From this analysis, we conclude that the analysis in [3] is incorrect. Their Eq. (24) does not remove the $j$ summation of their Eq. (22), for which reason the argumentation to move from Eq. (21) to Eq. (25) through Eqs. (22)-(24) in [3] is incorrect. Only noise sources that are uncorrelated in space, time, and vector orientation lead to Eq. (8). Using this analysis would give the correct interpretation for Eq. (25) in [3], which is then the same as Eq. (8) here. This is the second important correction of Eq. (25) in [3]. If we now take the receivers in $\boldsymbol{r}_{A}$ and $\boldsymbol{r}_{B}$ in the same direction $s_{j}$ and use that in Eq. (8) we obtain

$$
\boldsymbol{s} \cdot\left[\overline{\boldsymbol{h}}_{A}^{*}\left(\boldsymbol{r}_{B}\right)-\overline{\boldsymbol{h}}_{B}\left(\boldsymbol{r}_{A}\right)\right]|f(\omega)|^{2}=i \omega \mu\left\langle\left[\boldsymbol{s} \cdot \boldsymbol{h}\left(\boldsymbol{r}_{A}\right)\right]\left[\boldsymbol{s} \cdot \boldsymbol{h}^{*}\left(\boldsymbol{r}_{B}\right)\right]\right\rangle,
$$

which is written in the notation of [3] for direct comparison. This equation is correct only when used in combination with noise source specified above. Notice that the noise source correlation strength is proportional to the magnetic permeability, which is a propagation property of the medium. This is different than the Rytov theory for thermal electromagnetic radiation, where the noise source correlation strength is proportional the dissipation properties of the medium.

Equation (7) is a low-frequency approximation to the Green's function retrieval formulation, for which we should start with Eq. (3) with a minus sign. The result is

$$
\begin{aligned}
\operatorname{Im}\left\{G_{k r}\left(\boldsymbol{r}_{A}, \boldsymbol{r}_{B}\right)\right\}= & -\int_{\mathbb{D}} \operatorname{Im}\{\zeta\} G_{k p}^{*}\left(\boldsymbol{r}_{B}, \boldsymbol{r}\right) G_{r p}\left(\boldsymbol{r}_{A}, \boldsymbol{r}\right) \mathrm{d}^{3} \boldsymbol{r} \\
& +\int_{\mathbb{D}} \frac{\operatorname{Im}\{\eta\}}{|\eta|^{2}}\left[\epsilon_{j n q} \partial_{n} G_{k q}^{*}\left(\boldsymbol{r}_{B}, \boldsymbol{r}\right)\right] \\
& \times\left[\epsilon_{j m p} \partial_{m} G_{r p}\left(\boldsymbol{r}_{A}, \boldsymbol{r}\right)\right] \mathrm{d}^{3} \boldsymbol{r}
\end{aligned}
$$

from which it is observed that the second integral on the right-hand side contains equivalent electric dipole source contributions, because the curl operators act on the source position $\boldsymbol{r}$. Equation (10) is the generally valid Lagrangian form of magnetic field Green's function retrieval.

By the electromagnetic equivalence principle, we obtain the dual form for the electric field Green's function. Using the equivalence principle, we replace $\boldsymbol{h}$ by $-\boldsymbol{e}$, where $\boldsymbol{e}$ denotes the electric field, and we interchange $\eta$ and $-\zeta$ and interchange $\boldsymbol{j}^{e}$ and $\boldsymbol{j}^{m}$. We can then follow all the steps that have led to Eq. (10). This is equivalent to replacing the Green's function representing the magnetic field generated by magnetic-currenttype sources $G_{k r}\left(\boldsymbol{r}_{A}, \boldsymbol{r}_{B}\right)$ by the Green's function representing the electric field generated by electric-current-type sources with a minus sign $-\mathcal{G}_{k r}\left(\boldsymbol{r}_{A}, \boldsymbol{r}_{B}\right)$ and interchanging $\eta$ and $-\zeta$ to arrive at

$$
\begin{aligned}
\operatorname{Im}\left\{\mathcal{G}_{k r}\left(\boldsymbol{r}_{A}, \boldsymbol{r}_{B}\right)\right\}= & -\int_{\mathbb{D}} \operatorname{Im}\{\eta\} \mathcal{G}_{k p}^{*}\left(\boldsymbol{r}_{B}, \boldsymbol{r}\right) \mathcal{G}_{r p}\left(\boldsymbol{r}_{A}, \boldsymbol{r}\right) \mathrm{d}^{3} \boldsymbol{r} \\
& +\int_{\mathbb{D}} \frac{\operatorname{Im}\{\zeta\}}{|\zeta|^{2}}\left[\epsilon_{j n q} \partial_{n} \mathcal{G}_{k q}^{*}\left(\boldsymbol{r}_{B}, \boldsymbol{r}\right)\right] \\
& \times\left[\epsilon_{j m p} \partial_{m} \mathcal{G}_{r p}\left(\boldsymbol{r}_{A}, \boldsymbol{r}\right)\right] \mathrm{d}^{3} \boldsymbol{r}
\end{aligned}
$$

Observe that in the first and second integrals in the right-hand side of Eq. (11) the sources are electric and magnetic dipole sources, respectively. In the low frequency $\operatorname{limit} \operatorname{Im}\{\eta\} \approx 0$, we can now neglect the first integral in the right-hand side of Eq. (11). This means that the Green's function of the electric field generated by electric dipoles is extracted from correlating electric fields generated by noise sources of the magnetic current type only.

\section{VALIDATION EXAMPLE}

To illustrate the argument for the necessity of all source components for Green's function extraction, let us take the simplest example possible. Complexity of the configuration is not an issue, so the homogeneous full space example will suffice, and $\zeta=-\zeta^{*}$. The left-hand side of Eq. (7) for a homogeneous space is given by

$$
\begin{aligned}
\operatorname{Im}\left\{G_{k r}\left(\boldsymbol{r}_{A}, \boldsymbol{r}_{B}\right)\right\}= & \frac{1}{2}\left(\eta G_{A B}-\eta^{*} G_{A B}^{*}\right) \mathcal{I} \\
& -(2 \zeta)^{-1} \nabla_{A} \nabla_{A}\left(G_{A B}+G_{A B}^{*}\right),
\end{aligned}
$$

where $\mathcal{I}$ denotes the unit $3 \times 3$ matrix, $\nabla_{A}$ is the gradient acting on $\boldsymbol{r}_{A}$, and $G_{A B}$ denotes the homogeneous space scalar Green's function

$$
G_{A B}=\frac{\exp \left(-\sqrt{\eta \zeta}\left|\boldsymbol{r}_{A}-\boldsymbol{r}_{B}\right|\right)}{4 \pi\left|\boldsymbol{r}_{A}-\boldsymbol{r}_{B}\right|}
$$

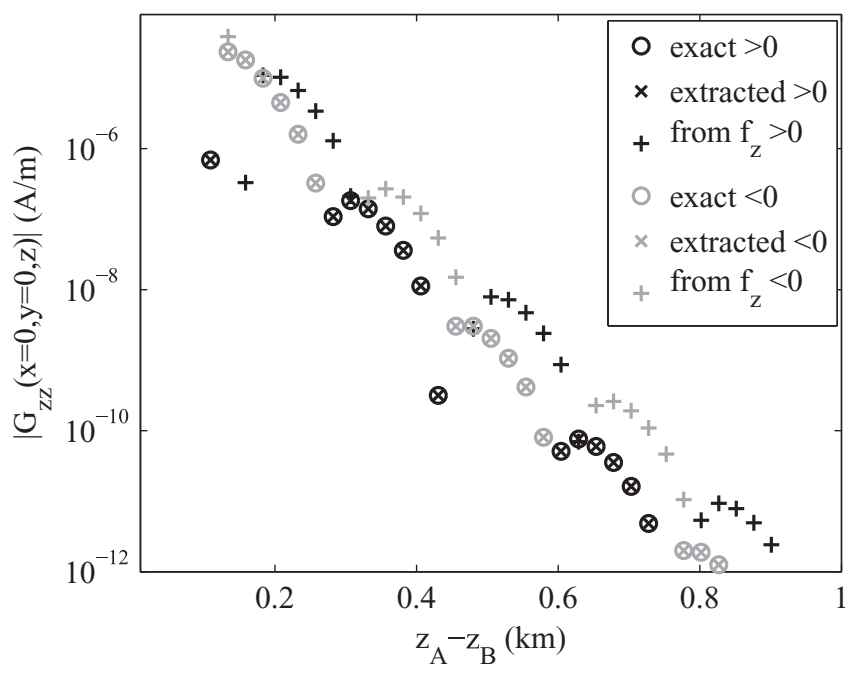

FIG. 1. Imaginary part of the vertical magnetic field generated by a vertical magnetic dipole as a function of vertical distance between the locations $\boldsymbol{r}_{A}$ and $\boldsymbol{r}_{B}$. 
The integral in the right-hand side of Eq. (7) is not very difficult to evaluate for a homogeneous space and given by

$$
\begin{aligned}
& -\operatorname{Im}\{\zeta\} \int_{\mathbb{D}} G_{k p}^{*}\left(\boldsymbol{r}_{B}, \boldsymbol{r}\right) G_{r p}\left(\boldsymbol{r}_{A}, \boldsymbol{r}\right) \mathrm{d}^{3} \boldsymbol{r} \\
& =\frac{\eta \eta^{*}}{\eta+\eta^{*}}\left(G_{A B}-G_{A B}^{*}\right) \mathcal{I}-\zeta^{-1} \nabla_{A} \nabla_{A} \frac{\eta^{*} G_{A B}+\eta G_{A B}^{*}}{\eta+\eta^{*}} .
\end{aligned}
$$

In the quasistatic limit where $\eta \approx \eta^{*}$, the right-hand sides of Eqs. (12) and (14) are equal. It is clear that this result can be obtained only when the summation over the subscript ${ }_{p}$ is taken for all values $p=1,2,3$. The configuration used in [3] has the two locations $\boldsymbol{r}_{A}$ and $\boldsymbol{r}_{B}$ in a homogeneous layer characterized by $\sigma=1 \mathrm{~S} / \mathrm{m}$ and magnetic permeability of free space. We use the same values and $\varepsilon=17 \varepsilon_{0}$, with $\varepsilon_{0}$ being the free space electric permittivity. The vertical component of the magnetic field generated by a vertical magnetic dipole is extracted from a summation of correlations of recorded vertical magnetic fields generated by vertical components of magnetic noise sources. The frequency of operation is taken as $100 \mathrm{~Hz}$. In Fig. 1, the right-hand side of Eq. (12), with $k=3$ and $r=3$, is shown as the exact solution in circles, and right-hand side of Eq. (14) with crosses, together with the results of the right-hand side of Eq. (25) in [3], shown with plus signs. The imaginary part of each field is shown, with the positive values using black markers and the negative field values using gray markers. It can be seen the errors are large and increasing with vertical source-receiver separation when only vertically aligned noise sources are used.

\section{CONCLUDING REMARKS}

Contrary to what is described in [3], the choice of the field type used in Green's function retrieval does not determine what the (noise) source characteristics should be. When the energy form is used, both electric and magnetic fields can be retrieved from correlations of field fluctuations generated by natural noise sources, such as thermal noise, that are proportional to the dissipative properties of the medium. When the Lagrangian form is used, both electric and magnetic fields in the quasistatic limit can be retrieved from correlations of field fluctuations generated by a volume distribution of uncorrelated sources of the magnetic current type, and whose correlation strengths are proportional to the magnetic permeability of the medium. Whether the retrieved Green's functions are a sum or difference of the Green's function and its complex conjugate counterpart depends on the definition of the Green's function. If for a particular definition the sum is obtained in the energy form, the Lagrangian form will retrieve the difference.

Contrary to what is used in [3], unidirectional (noise) sources are generally not sufficient for retrieving the Green's function, but all source components are necessary. When these are noise sources, these should be uncorrelated in their vector orientation as well as spatially and temporally.

\section{ACKNOWLEDGMENTS}

This work is part of the research program of the Netherlands research center for Integrated Solid Earth Science (ISES).
[1] K. Wapenaar, E. Slob, and R. Snieder, Phys. Rev. Lett. 97, 234301 (2006).

[2] R. Snieder, K. Wapenaar, and U. Wegler, Phys. Rev. E 75, 036103 (2007),

[3] S. Shamsalsadati and C. J. Weiss, Phys. Rev. E 81, 036603 (2010).

[4] R. Snieder, E. Slob, and K. Wapenaar, New J. Phys. 12 (2010).

[5] E. Slob and K. Wapenaar, Geophys. Res. Lett. 34, L05307 (2007).

[6] E. Slob and K. Wapenaar, Near Surface Geophysics 6, 391 (2008).
[7] S. M. Rytov, Y. A. Kravtsov, and V. I. Tatarskii, Principles of Statistical Radiophysics III (Springer, Berlin, 1989), Vol. 3.

[8] K. Wapenaar, Phys. Rev. Lett. 93, 254301 (2004).

[9] R. Snieder, Phys. Rev. E 69, 046610 (2004).

[10] R. Snieder, J. Sheiman, and R. Calvert, Phys. Rev. E 73, 066620 (2006).

[11] R. Snieder, Phys. Rev. E 74, 046620 (2006).

[12] J. T. Fokkema and P. M. van den Berg, Seismic Applications of Acoustic Reciprocity (Elsevier, Amsterdam, 1993). 